\title{
Effects of Induction of Labor on Maternal and Perinatal Outcome in Postdated Nulliparous Pregnancy
}

\author{
Shreyasi Karmakar ${ }^{1}$, Sabyasachi Bid², Tapan K Maiti
}

\begin{abstract}
Objective: This study aimed at determining the mode of delivery, maternal, and perinatal outcomes of induction of labor (IOL) in postdated pregnancies.

Methods: It was a prospective observational study. Nulliparous women with uncomplicated singleton pregnancies who have crossed their estimated date of delivery (EDD) were included in the study. Inclusion and exclusion criteria were strictly followed. A total of 280 patients were divided into study and control groups. Those who underwent IOL immediately after EDD were compared with women who were managed expectantly up to 42 weeks.

Results: The study group and control group consisted of 150 and 130 women, respectively. The rate of cesarean section (CS) (64.62\%) was significantly higher in an expectantly managed group. The most common indication of the CS was fetal distress in both groups, and the occurrence of oligohydramnios was quite higher in the control group. The rate of shoulder dystocia, cervical tear, and third-and fourth-degree perineal tear was significantly more in an expectantly managed group. Fetal distress was also more in that group.

Conclusion: Our study suggests that labor should be induced after crossing EDD if gestational age is confirmed so that the incidence of maternal and perinatal complications can be brought down to an acceptable level.

Keywords: Cesarean section, Induction of labor, Maternal complications, Perinatal complications, Postdated pregnancy.

Journal of South Asian Federation of Obstetrics and Gynaecology (2021): 10.5005/.jp-journals-10006-1870
\end{abstract}

\section{INTRODUCTION}

Postdated pregnancy is defined as a pregnancy that has extended beyond the estimated date of delivery (EDD) - American College of Obstetrics and Gynecology, 2004. ' The terms prolonged pregnancy, postdated, and postdatism are used to describe the same condition.

The incidence of postdated pregnancy is about 3 to $12 \%$ of all pregnancies. The common cause of prolonged pregnancy is inaccurate dating. ${ }^{2}$ Clinical methods that are used to confirm the period of gestation are last menstrual period, uterine size as estimated by bimanual examination in the first trimester, time of the first perception of fetal movements, and symphysis fundal height in a singleton pregnancy. Risk factors for postdatism are primiparity, history of previous post-term pregnancy, ${ }^{3}$ male fetuses, ${ }^{4}$ obesity, ${ }^{5}$ hormonal factors, and genetic predisposition. ${ }^{6}$

Post-term pregnancies are related to high fetal and neonatal morbidity and mortality as well as maternal morbidity. The perinatal mortality (stillbirths plus early neonatal deaths) at 42 weeks of gestation is double as compared to that at term (4-7 vs 2-3 per 1000 deliveries, respectively). ${ }^{7}$ It is thought that uteroplacental insufficiency, meconium aspiration, and intrauterine infection are the main causes of the increased perinatal death in these cases. ${ }^{8}$ Fetal morbidities like the passage of meconium, meconium aspiration syndrome (MAS), macrosomia, and dysmaturity are increased in post-term pregnancies. Post-term pregnancy itself leads to low umbilical cord pH levels (neonatal acidemia), low 5-minute Apgar scores, neonatal encephalopathy, and infant death. ${ }^{7}$

Post-term pregnancy may cause significant risks to the mother also. There is a high chance of: (1) labor dystocia (9-12\% vs $2-7 \%$ at term); (2) major degree of perineal lacerations (third- and fourth-degree tears), due to macrosomia (3.3\% vs $2.6 \%$ at term); (3) instrumental vaginal delivery; and (4) doubling in cesarean section
${ }^{1}$ Department of Obstetrics and Gynecology, ESI-PGIMSR, ESIC Medical College, Joka, Kolkata, India

${ }^{2}$ Department of Anaesthesiology and Critical Care, ESI-PGIMSR, ESIC Medical College, Joka, Kolkata, India

${ }^{3}$ Department of Obstetrics and Gynecology, N.R.S. Medical College and Hospital, Kolkata, India

Corresponding Author: Shreyasi Karmakar, Department of Obstetrics and Gynecology, ESI-PGIMSR, ESIC Medical College, Joka, Kolkata, India, Phone: +91 9830525422, e-mail: drshreyasibid@gmail.com

How to cite this article: Karmakar S, Bid S, Maiti TK. Effects of Induction of Labor on Maternal and Perinatal Outcome in Postdated Nulliparous Pregnancy. J South Asian Feder Obst Gynae 2021;13(2):106-110.

Source of support: Nil

Conflict of interest: None

(CS) rates ( $14 \%$ vs $7 \%$ at term). Cesarean delivery itself leads to a higher incidence of endometritis, postpartum hemorrhage, and thromboembolic diseases. ${ }^{9}$ In most of the situation, prolonged pregnancy causes mental anxiety to the mother, which should not be underestimated.

Prevention of post-term pregnancies is the first step of management. Induction of labor (IOL) at term is a good option. However, the risk of CS is increased with IOL. So, to avoid induction and expedite the spontaneous onset of labor at term, different types of minimally invasive interventions have been recommended, like membrane sweeping, ${ }^{10}$ unprotected sexual intercourse, ${ }^{11}$ nipple stimulation, ${ }^{12}$ and acupuncture. ${ }^{13}$

Women who have crossed their EDD but have not reached 42 weeks of gestation should be proposed for intensified antenatal

(0) Jaypee Brothers Medical Publishers. 2021 Open Access This article is distributed under the terms of the Creative Commons Attribution 4.0 International License (https://creativecommons.org/licenses/by-nc/4.0/), which permits unrestricted use, distribution, and non-commercial reproduction in any medium, provided you give appropriate credit to the original author(s) and the source, provide a link to the Creative Commons license, and indicate if changes were made. The Creative Commons Public Domain Dedication waiver (http://creativecommons.org/publicdomain/zero/1.0/) applies to the data made available in this article, unless otherwise stated. 
fetal monitoring to improve perinatal outcomes. Options for assessing fetal well-being include nonstress testing [cardiotocography (CTG)], biophysical scoring (BPP), modified BPP [CTG and amniotic fluid index (AFI)], contraction stress testing, and a combination of these modalities. There are so many techniques for fetal monitoring, and no single method has been found to be superior. ${ }^{2}$ Twice weekly antenatal fetal surveillance is widely practiced though there is no strict recommendation based on published research. ${ }^{1}$ CTG and [amniotic fluid (AFI)] volume estimations are an acceptable standard modality of fetal surveillance.

$\mathrm{IOL}$ is done when the benefits of delivery outweigh the risks associated with the continuation of pregnancy. The main problem of IOL in post-term, low-risk pregnancies is uterine overstimulation, fetal distress, failure of induction, and increased CS rates. There is also an increased risk of uterine rupture in women with previous CS. IOL is more likely to be successful when the cervix is favorable. Different techniques can be applied to assess cervical favorability and to predict the likelihood of success of IOL. These include digital cervical examination (Bishop score), ultrasonography measurement of cervical length, ${ }^{14}$ and recently some biochemical markers (maternal serum oestriol-to-estradiol ratio). ${ }^{15}$

Several studies have used prostaglandins for labor induction in post-term pregnancies, but no standardized regime has been established so far. Higher doses of the Prostaglandins (especially PGE1) increase the risk of uterine tachysystole and hyper-stimulation, leading to non-reassuring fetal CTG. PGE1 or misoprostol is not yet approved for IOL by the Drug Controller General of India. PGE2 or dinoprostone gel or suppository is commonly used for cervical ripening and IOL in unfavorable cervix. Low dose oxytocin infusion, transcervical Foley catheter, membrane sweeping, hygroscopic dilators (laminaria tent), mifepristone, no donors, relaxin, and hyaluronidase are presently not recommended for IOL in view of the nonavailability and lowquality evidence for their use. Intravenous oxytocin with or without artificial rupture of the membrane can be used for induction with a favorable cervix.

With this background, the present study was undertaken to compare the outcome of pregnancies that crossed EDD, and IOL was done.

\section{Objectives of the Study:}

- To study the maternal and perinatal outcomes in postdated nulliparous women who underwent IOL.

- To study the maternal and perinatal outcomes in postdated nulliparous women who were observed for spontaneous onset of labor up to 2 weeks past EDD.

- To compare the maternal and perinatal outcomes between the two groups.

\section{Materials and Methods}

This prospective observational study was conducted in the Department of Obstetrics and Gynecology, IPGMER and SSKM Hospital, Kolkata, in 2016, for 2 years duration. A total of 280 patients was selected from the maternity ward and labor room and divided into study group and control group.

\section{Inclusion Criteria}

- Nulliparous women

- Singleton pregnancy

- Gestational age $\geq 40$ weeks.

\section{Exclusion Criteria}

- Congenital anomaly of a fetus.

- Polyhydramnios

- Eclampsia.

- Antepartum hemorrhage

- Heart disease

- Elderly primigravida

- Medical disorder of pregnancy

- Postcesarean pregnancy.

The study group comprised of postdated antenatal women (as per inclusion and exclusion criteria), and elective IOL was done after crossing their EDD. The control group comprised of women who have crossed EDD, and expectant management was done till 42 weeks (42 weeks +0 days). If these women do not go into labor spontaneously, IOL was done. The expectant group may, therefore, went into spontaneous labor, elective lower uterine segment cesarean section (LUCS), or eventually ended up with an induced labor at $42+0$ weeks.

\section{Gestational Week}

We have followed the commonly accepted induction methods: In cases of unfavorable cervix, induction agent was intracervical PGE2 (dinoprostone) gel, while in cases of favorable cervix, the induction was done by oxytocin infusion or amniotomy followed by oxytocin infusion.

Every labor was monitored closely in all the patients of the study and control groups. The following maternal parameters were studied: Normal vaginal delivery (NVD), instrumental vaginal delivery, CS, uterine tachysystole, precipitate labor, postpartum hemorrhage $(\mathrm{PPH})$, prolonged labor, shoulder dystocia, retained placenta, uterine rupture, and maternal death. Neonatal parameters included in our study were fetal distress, stillborn, neonatal asphyxia, low birth weight (LBW), perinatal morbidities due to instrumental deliveries, MAS, neonatal sepsis, and neonatal death.

The study was accepted by the institutional ethical committee. Consent of all the patients in the study and control groups was taken. A proforma was formulated and filled for all the patients.

All the data were analyzed, plotted, and compared by using Student's t-test, chi-square test, and Fischer's exact test as appropriate.

\section{Results}

We have identified 280 nulliparous women as per inclusion criteria, and then randomization was done. One hundred and fifty women were induced electively after crossing EDD, and the rest one hundred and thirty were managed conservatively till 42 weeks.

Table 1 shows among 130 women in the expectant management group, only 67 (51.54\%) went to spontaneous labor, 60 (46.15\%) were induced for labor, and the rest 3 (2.31\%) were delivered by elective CS due to fetal indications.

Table 2 demonstrates the mode of delivery in the two groups. The mode of delivery was significantly different between the two

Table 1: Outcome of the women of an expectantly managed group $(n=130)$

\begin{tabular}{lc}
\hline Labor induction & $60(46.15 \%)$ \\
Spontaneous onset of labor & $67(51.54 \%)$ \\
Elective LUCS & $3(2.31 \%)$ \\
\hline
\end{tabular}


The Effects of Induction of Labor on Maternal and Perinatal Outcome

Table 2: Comparison of mode of delivery in study and control groups

\begin{tabular}{llll}
\hline Mode of delivery & Study $(n=150)$ & Control $(n=130)$ & $P$-value \\
\hline NVD & $70(46.67 \%)$ & $30(23.08 \%)$ & 0.000 \\
Instrumental delivery & $26(17.33 \%)$ & $16(12.31 \%)$ & \\
LUCS & $54(36 \%)$ & $84(64.61 \%)$ & \\
\hline
\end{tabular}

Table 3: Comparison of indications of CS in study versus control group

\begin{tabular}{lcll}
\hline Indication of LUCS & Study $(n=54)$ & Control $(n=84)$ & $p$-value \\
\hline Fetal distress & $18(33.33 \%)$ & $22(26.19 \%)$ & 0.36 \\
Oligohydramnios & $2(3.7 \%)$ & $15(17.86 \%)$ & 0.013 \\
Induction failure & $9(16.67 \%)$ & $20(23.81 \%)$ & 0.312 \\
NPOL & $15(27.78 \%)$ & $13(15.48 \%)$ & 0.080 \\
Thick meconium- & $10(18.51 \%)$ & $11(13.09 \%)$ & 0.384 \\
stained liquor & & & \\
Elective LUCS & 0 & $3(3.57 \%)$ & Not done \\
\hline
\end{tabular}

Table 4: Comparison of maternal complications in study versus control group

\begin{tabular}{lcll}
\hline Maternal complications & Study $(n=150)$ & Control $(n=130)$ & $p$-value \\
\hline Shoulder dystocia & $10(6.66 \%)$ & $18(13.84 \%)$ & 0.045 \\
Prolonged labor & $24(16 \%)$ & $25(19.23 \%)$ & 0.477 \\
PPH & $5(3.33 \%)$ & $10(7.69 \%)$ & 0.105 \\
$3^{\circ}$ and $4^{\circ}$ perineal tear & $5(3.33 \%)$ & $15(11.53 \%)$ & 0.007 \\
Cervical tear & $2(1.33 \%)$ & $9(6.92 \%)$ & 0.016 \\
Puerperal sepsis & $1(0.66 \%)$ & $3(2.30 \%)$ & 0.250 \\
\hline
\end{tabular}

groups ( $p<0.05$ ). The rate of normal vaginal delivery was quite higher in the induction group than that in the expectantly managed group ( $46.67 \%$ vs $23.08 \%)$. On the contrary, the rate of CS was significantly higher in the expectant management group (64.61\%) compared to the study group (36\%). The incidence of instrumental vaginal delivery was not significantly different in the two groups. It was $17.33 \%$ in the study group and $12.31 \%$ in the control group.

The indication of CS is listed in Table 3. The most common indication of LUCS was fetal distress in both the groups $(33.33 \%$ in IOL group and26.19\%in expectant group). In the list, we can see that the risk of oligohydramnios and its indication for LUCS was significantly higher in the expectantly managed group $(3.7 \%$ in IOL and $17.86 \%$ in expectant). Three women in the expectant management group were delivered by elective LUCS due to oligohydramnios and macrosomic baby.

Table 4 describes various maternal complications in two groups. The analysis showed the rate of shoulder dystocia was much lower in the induction group (6.66\%) compared to the expectantly managed group (13.84\%). The $p$ value was $<0.05$. While analyzing various types of genital injury during vaginal delivery, it was found that the occurrence of third- and fourth-degree perineal tear (3.33\% in IOL vs $11.53 \%$ in expectant, $p$-value- 0.007 ) and cervical tear (1.33\% in IOL vs6.92\% in expectant, $p$-value-0.016) was statistically significantly higher in the expectantly managed group.

Table 5 showed that fetal distress was significantly higher in the expectantly managed group ( $14.66 \%$ in IOL vs $26.92 \%$ in expectant group, $p$-value $=0.011$ ). The risk of stillborn fetus, birth asphyxia, birth injury, MAS, and neonatal sepsis in our study was lower in IOL group although the difference was not statistically significant.
Table 5: Comparison of perinatal complications in study versus control group

\begin{tabular}{lcll}
\hline Fetal complications & Study $(n=150)$ & Control $(n=130)$ & $p$-value \\
\hline Fetal distress & $22(14.66 \%)$ & $35(26.92 \%)$ & 0.011 \\
Stillborn & $1(0.66 \%)$ & $2(1.53 \%)$ & 0.477 \\
Birth asphyxia & $1(0.66 \%)$ & $4(3.07 \%)$ & 0.128 \\
LBW & $1(0.66 \%)$ & 0 & Not done \\
Birth injury & $2(1.33 \%)$ & $5(3.84 \%)$ & 0.180 \\
MAS & $5(3.33 \%)$ & $8(6.15 \%)$ & 0.262 \\
Neonatal sepsis & 0 & $2(1.53 \%)$ & Not done \\
Neonatal death & $8(5.33 \%)$ & $4(3.07 \%)$ & 0.352 \\
\hline
\end{tabular}

Table 6: Comparison of birth weight in study versus control

\begin{tabular}{lccl}
\hline Weight $(\mathrm{kg})$ & Study $(n=150)$ & Control $(n=130)$ & $p$-value \\
\hline$<2.5$ & $1(0.66 \%)$ & 0 & 0.196 \\
$2.5-4$ & $146(97.33 \%)$ & $123(94.61 \%)$ & \\
$>4$ & $3(2 \%)$ & $7(5.38 \%)$ & \\
\hline
\end{tabular}

Table 6 depicts the comparison of birth weight of neonates. Although labor induction at 40 weeks was associated with a reduced chance to develop a macrosomic baby, these data were not statistically significant.

\section{Discussion}

One of the most important findings in our study, a higher risk of CS in the expectantly managed group, is in accordance with other studies. The ARRIVE Trial ${ }^{16}$ was a randomized control trial where 6106 low-risk nulliparous women were recruited and were induced at 39 weeks and were compared with those who chose for expectant management till 42 weeks. There was a decrease in the incidence of CS with induction at 39 weeks (18\% vs $22 \%$, RR 0.84). Several studies indicated a lower risk of CS at 40 or 41 weeks compared to expectant management. ${ }^{17,18}$ Although few studies have indicated a higher likelihood of CS following labor induction, primiparous subgroup in their study found a similar trend of results (as our study) concerning the mode of delivery and risk of CS as compared to the whole study group. ${ }^{19-21}$ Preinduction Bishop's score, body mass index, and age of the patient are some confounding factors that may influence the results in different study groups, but overall most of the studies are in conclusion that primiparous women with expectant management beyond 41 weeks are at increased risk of cesarean delivery. These conclusions are in line with our study.

In our study, the commonest indication of CS in both the group was fetal distress $(33.33 \%$ and $26.19 \%$, respectively), but here the difference between the two groups is not statistically significant. Incidence of fetal distress substantially increases after 40 weeks of gestation due to uteroplacental insufficiency and meconium passage. ${ }^{8}$ One of the major complications of IOL is uterine hyperstimulation and thick meconium-stained liquor. ${ }^{22}$ Our study also reveals the same fact.

Secondly, we noted that the risk of developing oligohydramnios was more in an expectantly managed group compared to the labor induction group. The difference between the two groups is statistically significant. Oligohydramnios is an independent risk factor of prolonged pregnancy. Meconium release into an already 
reduced liquor volume results in thick meconium and MAS of a fetus. ${ }^{23,24}$ Our study does not show a significant increase in the number of meconium-stained liquor as an indication of CS.

Regarding maternal complications, our study shows that the expectantly managed group had a significantly higher rate of shoulder dystocia, third- and fourth-degree perineal tear, and cervical tear. Although the chances of prolonged labor, PPH, and puerperal sepsis were more in the aforementioned group than that in the induced labor group, the result is imprecise, not statistically significant. Other studies, like Treger et al. and Rand et al., have also found that the incidence of macrosomia and as a result perineal tear are more in late-term pregnancy. ${ }^{25,26}$ In our study, increased shoulder dystocia in the expectant management group is mainly due to a big size baby. Perineal and cervical tears were also caused during the delivery of a big-sized baby. There are other studies that have not shown a significant difference in the mode of delivery and maternal complications in both groups of women. ${ }^{27,28}$ Their results were probably influenced by the parity of the patients. Induction in multiparous women has more favorable outcome with respect to normal vaginal delivery, and so, the rate of postdelivery complications is also less. We have omitted this biasness by excluding the multiparous women from our study.

While analyzing the fetal complications, our study shows that the rate of fetal distress was significantly more in an expectantly managed group than that in the induction group. Here, prolonged duration of pregnancy leading to oligohydramnios and cord compression was the causes of fetal distress. IOL has not contributed much to the occurrence of fetal distress. There are mixed supportive data in this regard. Some studies have shown that induction parse is responsible for fetal distress. ${ }^{22}$ On the contrary, most of the studies have concluded that advanced gestational age ( $>40$ weeks) is at high risk of developing fetal distress due to decreased volume of liquor amnii, umbilical cord compression, and thick meconium in liquor. ${ }^{29,30}$ Present study also showed a higher rate of MAS, but it is not statistically significant. In this context, we would like to mention that method of labor induction also has an influence on fetomaternal complications. Misoprostol (PGE1) administered vaginally causes meconium staining of liquor particularly in unfavorable cervix and MAS in the fetus. ${ }^{31,32}$ Christensen et al. demonstrated that the combination of oxytocin induction, preceded by dinoprostone application, is safe and significantly shortens induction delivery interval. ${ }^{33}$ Analysis of birth weight did not show any statistically significant difference while comparing the two groups. This result is in accordance with other studies. ${ }^{34}$

By analyzing all the differences between the two groups, it must be kept in mind that a higher gestational age is associated with an increasing rate of fetomaternal complications. Alexander et al. observed a higher rate of labor complications, such as prolonged duration of labor. ${ }^{35}$ Furthermore, the risk of maternal infections, $\mathrm{PPH}$, and obstetric trauma increases with gestational age. ${ }^{30,36,37}$ There is also an increased risk of CS with higher gestational age. ${ }^{30,38}$ Present study found that the risk of fetal distress rises strongly with gestational age. So, it should be emphasized that higher gestational age is associated with a rise in stillbirth, perinatal or neonatal deaths, meconium aspiration, pneumonia, or asphyxia as evidenced by other studies.

\section{Conclusion}

The aim of this study was to compare the effect of routine labor induction immediately after EDD to the practice of waiting for spontaneous onset of labor until 2 weeks after EDD. We found that routine induction at $40+0$ to 6 weeks gestation is associated with a decreased risk of CS, oligohydramnios, shoulder dystocia, thirdand fourth-degree perineal tear, cervical tear, and fetal distress of newborn. Our study has strictly included the nulliparous women, which may be the reason of unfavorable maternal outcomes in cases of expectant management. Our findings do not support waiting for spontaneous onset of labor up to 2 weeks past EDD. It highlights the importance of discussing whether the risk related to the procedure of IOL outweighs the harm of ongoing pregnancy. It also gives some idea whether IOL should be applied to a larger population of low-risk women.

\section{Clinical Significance}

Any pregnancy that goes beyond 40 weeks should be considered as high risk. So, proper management should be done to decrease the rate of perinatal morbidity and mortality. The emotional impact as well as peripartum complications of a mother should also be taken into consideration while allowing a pregnancy to prolong. So, a policy should be made to intervene in pregnancy at an optimum gestational period so that a proper balance could be made between the risk of induction and that of ongoing pregnancy.

\section{References}

1. ACOG (American College of Obstetricians and Gynecologists). Management of postterm pregnancy. ACOG Practice bulletin no. 55. Obstet Gynecol 2004;104:639-646. DOI: 10.1097/00006250200409000-00052.

2. Crowley P. Interventions for preventing or improving the outcome of delivery at or beyond term (Cochrane review). In: The Cochrane library. Chicester: John Wiley \& Sons, Ltd., 2004.

3. Olesen AW, Basso O, Olsen J. An estimate of the tendency to repeat postterm delivery. Epidemiology 1999;10:468-469. DOI: 10.1097/00001648-199907000-00026.

4. Divon MY, Ferber A, Nisell $H$, et al. Male gender predisposes to prolongation of pregnancy. Am J Obstet Gynecol 2002;187:1081-1083. DOI: $10.1067 / \mathrm{mob} .2002 .126645$.

5. Stotland NE, Washington AE, Caughey AB. Pre-pregnancy body mass index and length of gestation at term. Am J Obstet Gynecol 2007;197:378.e1. DOI: 10.1016/j.ajog.2007.05.048.

6. Laursen M, Bille C, Olesen AW, et al. Genetic influence on prolonged gestation: a population-based Danish twin study. Am J Obstet Gynecol 2004;190:489-494. DOI: 10.1016/j.ajog.2003.08.036.

7. Hilder L, Costeloe K, Thilaganathan B. Prolonged pregnancy: evaluating gestation-specific risks of fetal and infant mortality. $\mathrm{Br}$ J Obstet Gynaecol 1998;105:169-173. DOI: 10.1111/j.1471-0528.1998. tb10047.x.

8. Hannah ME. Postterm pregnancy: should all women have labour induced? A review of the literature. Fetal Matern Med Rev 1993;5:3. DOI: https://doi.org/10.1017/S0965539500000681

9. Alexander JM, Mclntire DD, Leveno KJ. Prolonged pregnancy: induction of labour and caesarean births. Obstet Gynecol 2001;97:911. DOI: 10.1016/s0029-7844(01)01354-0.

10. Boulvain M, Stan C, Irion O. Membrane sweeping for induction of labour. Cochrane Database Syst Rev 2005;1:CD000451. DOI: 10.1002/14651858.CD000451.pub3.

11. Schaffir J. Survey of folk beliefs about induction of labor. Birth 2002;29:47-51. DOI: 10.1046/j.1523-536x.2002.00047.x.

12. Kavanagh J, Kelly AJ, Thomas J. Breast stimulation for cervical ripening and induction of labour. Cochrane Database Syst Rev 2005;3:CD003392. DOI: 10.1002/14651858.CD003392.pub2.

13. Smith CA, Crowther CA. Acupuncture for induction of labour. Cochrane Database Syst Rev 2004;1:CD002962. DOI: 10.1002/14651858. CD002962.pub2. 
14. Rozenberg $P$, Chevret $S$, Ville $Y$. Comparison of pre-induction ultrasonographic cervical length and Bishop score in predicting risk of caesarean section after labour induction with prostaglandins. Gynecol Obstet Fertil 2005;33:17-22. DOI: 10.1016/j.gyobfe.2004.11.006.

15. Torricelli M, Novembri R, Voltolini C, et al. Biochemical and biophysical predictors of the response to the induction of labour in nulliparous postterm pregnancy. Am J Obstet Gynecol 2011;204:39.e1. DOI: 10.1016/j.ajog.2010.08.014

16. Grobman WA, Rice MM, Reddy UM, et al. Labour induction versus expectant management in low risk nulliparous women. NEJM 2018;379(6):513-523. DOI: 10.1056/NEJMoa1800566.

17. Stock SJ, Ferguson E, Duffy A, et al. Outcomes of elective induction of labour compared with expectant management: population-based study. BMJ 2012;344:e2838. DOI: 10.1136/bmj.e2838.

18. Roach VJ, Rogers MS. Pregnancy outcome beyond 42 weeks gestation. Int J Gynecol Obstet 1997;59:19-24. DOI: 10.1016/s00207292(97)00179-3.

19. Wolfe H, Timofeev J, Tefera E, et al. Risk of caesarean in obese nulliparous women with unfavourable cervix: elective induction vs expectant management at term. Am J Obstet Gynecol 2014;211:530. DOI: 10.1016/j.ajog.2014.01.034.

20. Vrouenraets FP, Roumen FJ, Dehing CJ, et al. Bishop score and risk of caesarean delivery after induction of labour in nulliparous women. Obstet Gynecol 2005;105:690-697. DOI: 10.1097/01. AOG.0000152338.76759.38.

21. Prysak M. Elective induction versus spontaneous labour: a casecontrol analysis of safety and efficacy. Obstet Gynecol 1998;92:47-52. DOI: 10.1016/s0029-7844(98)00115-x.

22. WHO. WHO recommendation for induction of labour. Geneva: World Health Organisation, 2011.

23. Oz AU, Holub B, Mendilcioglu I, et al. Renal artery doppler investigation of the etiology of oligohydramnios in postterm pregnancy. Obstet Gynecol 2002;100:715. DOI: 10.1016/s0029-7844(02)02203-2.

24. Trimmer KJ, Leveno KJ, Peters MT, et al. Observation on the cause of oligohydramnios in prolonged pregnancy. Am J Obstet Gynecol 1990;163:1900. DOI: 10.1016/0002-9378(90)90771-x.

25. Treger M, Hallak M, Silberstein T, et al. Post-term pregnancy: should induction of labour be considered before 42 weeks? J Matern Fetal Neonatal Med 2002;11:50-53. DOI: 10.1080/jmf.11.1.50.53.

26. Rand L, Robinson JN, Economy KE, et al. Postterm induction of labour revited. Obstet Gynecol 2000;96:779-783. DOI: 10.1016/s00297844(00)01002-4.
27. Hutcheon JA, Harper S, Strumpf EC, et al. Using interinstitutional practice variation to understand the risk and benefits of routine labour induction at 41(+0) weeks. BJOG 2015;122:973-981. DOI: 10.1111/1471-0528.13007.

28. Bonder-Adler B, Bodner K, Pateisky N, et al. Influence of labour induction on obstetric outcomes in patients with prolonged pregnancy. Wien Klin Wchenschr 2005;117:287-292. DOI: 10.1007/ s00508-005-0330-2.

29. Bruckner TA, Cheng YW, Coughey AB. Increased neonatal mortality among normal weight births beyond 41 weeks of gestation in California. Am J Obster Gynecol 2008;199:4210-4219. DOI: 10.1016/j. ajog.2008.05.015.

30. Olesen AW, Westergaard JG, Olsen J. Perinatal and maternal complications related to postterm delivery: a national register-based study, 1978-1993. Am J Obstet Gynecol 2003;189:222-227. DOI: 10.1067/mob.2003.446.

31. Liu A, Lv J, Hu Y, et al. Efficacy and safety of intravaginal misoprostol versus intracervical dinoprostone for labour induction at term: a systemic review and meta-analysis. J Obstet Gynecol Res 2014;40(4):897-906. DOI: 10.1111/jog.12333.

32. Indira Mani Y, Ratnakumari V, Chaitanya Sravanthi TS. Induction of labour with vaginal misoprostol and incidence of meconium stained liquor and fetal outcome. JEBMH 2016;3(7):221-225.

33. Christensen FC, Tehranifar M, Gonzalez JL, et al. Randomized trial of concurrent oxytocin with a sustained-release dinoprostone vaginal insert for labour induction at term. Am J Obstet Gynecol 2002;186(1):61-65. DOI: 10.1067/mob.2002.118309.

34. Thangarajah F, Scheufen P, Kirn V, et al. Induction of labour in late and postterm pregnancies and its impact of maternal and neonatal outcome. Geburtshilfe Frauenheilkd. 2016;76(7):793-798. DOI: 10.1055/s-0042-107672.

35. Alexander JM, McIntire DD, Leveno KJ. Forty weeks and beyond: pregnancy outcomes by weeks of gestation. Obstet Gynecol 2000;96:291-294. DOI: 10.1016/s0029-7844(00)00862-0.

36. Wang M, Fontaine P. Common questions about late-term and postterm pregnancy. Am Fam Physician 2014;90:160-165.

37. Mandruzzato G, Alfirevic Z, Chervenak F, et al. Guidelines for management of postterm pregnancy. J Perinat Med 2010;38:111-119. DOI: 10.1515/jpm.2010.057.

38. Nakling J, Becke B. Pregnancy risk increases from 41 weeks of gestation. Acta Obstet Gynecol Scand 2006;85:663-668. DOI: 10.1080/00016340500543733. 